\title{
PeerJ
}

\section{An integrated pipeline for next generation sequencing and annotation of the complete mitochondrial genome of the giant intestinal fluke, Fasciolopsis buski (Lankester, 1857) Looss, 1899}

\author{
Devendra Kumar Biswal ${ }^{1}$, Sudeep Ghatani ${ }^{2}$, Jollin A. Shylla ${ }^{2}$, \\ Ranjana Sahu ${ }^{2}$, Nandita Mullapudi ${ }^{3}$, Alok Bhattacharya ${ }^{4}$ and \\ Veena Tandon ${ }^{1,2}$ \\ ${ }^{1}$ Bioinformatics Centre, North-Eastern Hill University, Shillong, Meghalaya, India \\ ${ }^{2}$ Department of Zoology, North-Eastern Hill University, Shillong, Meghalaya, India \\ ${ }^{3} \mathrm{M} / \mathrm{s}$ Genotypic Technologies, Bangalore, India \\ ${ }^{4}$ School of Life Sciences, Jawaharlal Nehru University, New Delhi, India
}

Submitted 5 September 2013

Accepted 21 October 2013

Published 12 November 2013

Corresponding authors

Alok Bhattacharya,

alok0200@mail.jnu.ac.in,

alok.bhattacharya@gmail.com

Veena Tandon,

tandonveena@gmail.com

Academic editor

Kenta Nakai

Additional Information and Declarations can be found on page 20

DOI 10.7717/peerj.207

Copyright

2013 Biswal et al.

Distributed under

Creative Commons CC-BY 3.0

OPEN ACCESS

\section{ABSTRACT}

Helminths include both parasitic nematodes (roundworms) and platyhelminths (trematode and cestode flatworms) that are abundant, and are of clinical importance. The genetic characterization of parasitic flatworms using advanced molecular tools is central to the diagnosis and control of infections. Although the nuclear genome houses suitable genetic markers (e.g., in ribosomal (r) DNA) for species identification and molecular characterization, the mitochondrial $(\mathrm{mt})$ genome consistently provides a rich source of novel markers for informative systematics and epidemiological studies. In the last decade, there have been some important advances in mtDNA genomics of helminths, especially lung flukes, liver flukes and intestinal flukes. Fasciolopsis buski, often called the giant intestinal fluke, is one of the largest digenean trematodes infecting humans and found primarily in Asia, in particular the Indian subcontinent. Next-generation sequencing (NGS) technologies now provide opportunities for high throughput sequencing, assembly and annotation within a short span of time. Herein, we describe a high-throughput sequencing and bioinformatics pipeline for $\mathrm{mt}$ genomics for $F$. buski that emphasizes the utility of short read NGS platforms such as Ion Torrent and Illumina in successfully sequencing and assembling the mt genome using innovative approaches for PCR primer design as well as assembly. We took advantage of our NGS whole genome sequence data (unpublished so far) for F. buski and its comparison with available data for the Fasciola hepatica mtDNA as the reference genome for design of precise and specific primers for amplification of mt genome sequences from F. buski. A long-range PCR was carried out to create an NGS library enriched in mt DNA sequences. Two different NGS platforms were employed for complete sequencing, assembly and annotation of the F. buski $\mathrm{mt}$ genome. The complete $\mathrm{mt}$ genome sequences of the intestinal fluke comprise $14,118 \mathrm{bp}$ and is thus the shortest trematode mitochondrial genome sequenced to date. The noncoding control regions are separated into two parts by the tRNA-Gly gene and don't contain either tandem repeats or secondary structures, which are 
typical for trematode control regions. The gene content and arrangement are identical to that of F. hepatica. The F. buski mtDNA genome has a close resemblance with F. hepatica and has a similar gene order tallying with that of other trematodes. The mtDNA for the intestinal fluke is reported herein for the first time by our group that would help investigate Fasciolidae taxonomy and systematics with the aid of mtDNA NGS data. More so, it would serve as a resource for comparative mitochondrial genomics and systematic studies of trematode parasites.

Subjects Bioinformatics, Evolutionary Studies, Genomics, Parasitology, Zoology

Keywords Fasciolopsis buski, Mitochondria, Next generation sequencing, Contigs

\section{INTRODUCTION}

Fasciolopsis buski, often called the giant intestinal fluke, is one of the largest digenean trematode flatworms infecting humans and found primarily in Asia and the Indian subcontinent, also occurring in Taiwan, Thailand, Laos, Bangladesh, India, and Vietnam. The trematode predominates in areas where pigs are raised, they being the most important reservoirs for the organism and where underwater vegetables viz. water chestnut, lotus, caltrop and bamboo are consumed. It is an etiological agent of fasciolopsiasis, a disease that causes ulceration, haemorrhage and abscess of the intestinal wall, diarrhoea, and even death if not treated properly. Interestingly, most infections are asymptomatic with high rates of infection (up to 60\%) in India and the mainland China (Le et al., 2004). Among animals, pigs are the main reservoir of $F$. buski infection. In India, the parasite has been reported from different regions including the Northeast and variations in the morphology of the fluke have been observed from different geographical regions (Roy \& Tandon, 1993). F. buski occurs in places with warm, moist weather and is the only single species in the genus found in aquatic environments. The complex life cycle combined with the specific immune evasion traits of parasites make research and drug or vaccine programs for intestinal flukes very difficult; consequently, new methods to control this parasite are required. Being one of the most important intestinal flukes from an epidemiological point of view, F. buski seeks considerable attention from the scientific community and the available gene sequences for the organism on the public domain remain scarce thereby restricting research avenues. Therefore, fasciopsiasis has become a public health issue and is of major socioeconomic significance in endemic areas.

Metazoan mitochondrial ( $\mathrm{mt}$ ) genomes, ranging in size from 14 to $18 \mathrm{~kb}$, are typically circular and usually encode 36-37 genes including 12-13 protein-coding genes, without introns and with short intergenic regions (Wolstenholme, 1992). Due to their maternal inheritance, faster evolutionary rate change, lack of recombination, and comparatively conserved genome structures mitochondrial DNA (mtDNA) sequences have been extensively used as molecular markers for studying the taxonomy, systematics, and population genetics of animals (Li et al., 2008; Catanese, Manchado \& Infante, 2010). At the time of 
writing this manuscript, quite a number of complete metazoan $\mathrm{mt}$ genomes are already deposited in GenBank (Benson et al., 2005) and other public domain databases viz. Mitozoa (D’Onorio de Meo et al., 2011), mainly for Arthropoda, Mollusca, Platyhelminthes, Nematoda, and Chordata (Chen et al., 2009). Presently, the class Trematoda comprises about 18,000 nominal species, and the majority of them can parasitize mammals including humans as their definitive host (Olson et al., 2003). Despite their medical and economical significance, most of them still remain poorly understood at the molecular level. In particular, the complete $\mathrm{mt}$ genomes of the species belonging to the family Fasciolidae are not at all available in the public domain. Complete or near-complete mt genomes are now available for 15 odd species or strains of parasitic flatworms belonging to the classes Trematoda and Cestoda. To date, a PCR-based molecular characterization using ITS1\&2 molecular markers for F. buski have been carried out (Prasad et al., 2007). However, further datasets generated by high-throughput sequencing and comparative transcriptome analysis could bring a more comprehensive understanding of the parasite biology for studying parasite-host interactions and disease as well as parasite development and reproduction, with a view towards establishing new methods of prevention, treatment or control.

Until quite recently, sequencing of $\mathrm{mt}$ genomes was somewhat challenging and a daunting task. It has been approached using the conventional strategy of combining long-range PCR with subsequent primer walking. The paradigm shift caused by the third generation sequencing technologies have paved the way for Next-Generation Sequencing (NGS) technologies, which encourages proposals for more straightforward integrated pipelines for sequencing complete mt genomes (Jex, Littlewood \& Gasser, 2010) that are more cost effective and less time consuming.

Here in, we present a straightforward approach for reconstructing novel mt genomes directly from NGS data generated from total genomic DNA extracts. We took advantage of the whole genome sequence data for F. buski (DK Biswal, S Ghatani, JA Shylla, R Sahu, N Mullapudi, A Bhattacharya, V Tandon, unpublished data), generated by NGS and its comparison with the existing data for the F. hepatica mt genome sequence to design precise and specific primers for amplification of $\mathrm{mt}$ genome sequences of F. buski. We then carried out long-range PCR to create a NGS library enriched in mt DNA sequences. We utilized two different next generation sequencing platforms to completely sequence the mitochondrial genome, and applied innovative approaches to assemble the mitochondrial genome in silico and annotate it. When verifying one region of the assembly by Sanger sequencing it was found to match our assembly results. The purpose of the present study was to sequence the mt genome of $F$. buski for the first time with a novel strategy, compare its sequences and gene organization, identify any adaptive mutations in the 12 protein-coding genes of the intestinal parasite species, and to reconstruct the phylogenetic relationships of several species of Trematoda and Cestoda in the Phylum Platyhelminthes, using mtDNA sequences available in GenBank. 


\section{MATERIALS \& METHODS}

\section{Parasite material and DNA extraction}

Live adult F. buski were obtained from the intestine of freshly slaughtered pig, Sus scrofa domestica at local abattoirs meant for normal meat consumption and not specifically for this design of study. The worms recovered from these hosts represented the geographical isolates from Shillong (coordinates $25.57^{\circ} \mathrm{N} 91.88^{\circ} \mathrm{E}$ ) area in the state of Meghalaya, Northeast India. Eggs were obtained from mature adult flukes by squeezing between two glass slides. For the purpose of DNA extraction, adult flukes collected from different host animals were processed singly; eggs recovered from each of these specimens were also processed separately. The adult flukes were first immersed in digestion extraction buffer [containing $1 \%$ sodium dodecyl sulfate (SDS), $25 \mathrm{mg}$ Proteinase $\mathrm{K}$ ] at $37^{\circ} \mathrm{C}$ for overnight. DNA was then extracted from lysed individual worms by standard ethanol precipitation technique (Sambrook, Fitsch \& Maniatis, 1989) and also extracted from the eggs on FTA cards using Whatman's FTA Purification Reagent. DNA was subjected to a series of enzymatic reactions that repair frayed ends, phosphorylate the fragments, and add a single nucleotide 'A' overhang and ligate adaptors (Illumina's TruSeq DNA sample preparation kit). Sample cleanup was done using Ampure XP SPRI beads. After ligation, $\sim 300-350 \mathrm{bp}$ fragment for short insert libraries and $\sim 500-550 \mathrm{bp}$ fragment for long insert libraries were size selected by gel electrophoresis, gel extracted and purified using Minelute columns (Qiagen). The libraries were amplified using 10 cycles of PCR for enrichment of adapter-ligated fragments. The prepared libraries were quantified using Nanodrop and validated for quality by running an aliquot on High Sensitivity Bioanalyzer Chip (Agilent). 2X KapaHiFiHotstart PCR ready mix (Kapa Biosystems Inc., Woburn, MA) reagent was used for PCR. The Ion torrent library was made using Ion Plus Fragment library preparation kit (Life Technologies, Carlsbad, US) and the Illumina library was constructed using TruSeqTM DNA Sample Preparation Kit (Illumina, Inc., US) reagents for library prep and TruSeq PE Cluster kit v2 along withTruSeq SBS kit v5 36 cycle sequencing kit (Illumina, Inc., US) for sequencing.

\section{Primer design strategy and Polymerase Chain Reaction (PCR)}

$\sim 16$ million 100 base-paired end reads were available for F. buski as a part of an independent attempt towards whole genome sequencing of $F$. buski. In order to recover mtDNA coding sequences from this data, Fasciola hepatica mt genome with accession AF216697.1 was retrieved from GenBank as a reference mt Genome and alignment using Bowtie (v2-2.0.0-beta6/bowtie2 -end-to-end -very-sensitive-no-mixed -phred64) (Langmead et al., 2009). In all, 1625 paired end reads were obtained, which were aligned to different intervals in the $F$. hepatica $\mathrm{mt}$ genome, covering $\sim 3 \mathrm{~kb}$ of the $14 \mathrm{~kb}$ F. hepatica $\mathrm{mt}$ genome. Accordingly, primers were designed at these regions, using sequence information from F. buski to ensure optimum primer designing as shown in Table 1. Long-range PCR was carried out using $10 \mathrm{ng}$ of genomic DNA from F. buski and the following PCR conditions: 10 ng of FD-2 DNA with $10 \mu \mathrm{M}$ Primer mix in $10 \mu \mathrm{l}$ reaction PCR cycling conditions $-98^{\circ} \mathrm{C}$ for $3 \mathrm{~min}, 35$ cycles of $98^{\circ} \mathrm{C}$ for $30 \mathrm{~s}, 60$ for $30 \mathrm{~s}, 72$ for $2 \mathrm{~min} 30 \mathrm{~s}$, 


\begin{tabular}{|c|c|c|c|c|}
\hline $\begin{array}{l}\text { Primer } \\
\text { name }\end{array}$ & $\begin{array}{l}\text { Primer } \\
\text { sequence }\end{array}$ & Product & $\begin{array}{l}\text { Expected } \\
\text { length }\end{array}$ & $\begin{array}{l}\text { Observed } \\
\text { length (bp) }\end{array}$ \\
\hline $\mathrm{F} 1$ & TACATGCGGATCCTATGG & $\mathrm{P} 1$ & 1525 & $500,700,1000$ \\
\hline $\mathrm{F} 2$ & AAAGACATACAAACAACAAC & & & \\
\hline F3 & TCTTTAGTGTATTCTTTGGGTCATG & $\mathrm{P} 2$ & 2660 & 3000 \\
\hline $\mathrm{F} 4$ & AАСАACСССААССТАСССТ & & & \\
\hline F5 & GTTTGTTGAGGGTAGGTTGGGG & P3 & 1623 & 1600 \\
\hline F6 & CAAATCATTAATGCGAGG & & & \\
\hline F7 & CTTTTTGATGCCTGTGTTCATAG & $\mathrm{P} 4$ & 2010 & 2000 \\
\hline F8 & АССТТТСАААСААТСССССА & & & \\
\hline F9 & CGGATTTATAGATGGTAGTGCCTG & P5 & 1037 & 1000 \\
\hline F10 & CCGGATATACACTAACAAACATAATTAAG & & & \\
\hline F11 & GTTTGTTAGTGTATATCCGGTTGAAG & P6 & 2361 & 2200 \\
\hline F12 & GGCAGCAACCAAAGTAGAAGA & & & \\
\hline F13 & TATTTCTTGGTTGTTGGAGGCTAT & P7 & 3783 & 4000,8000 \\
\hline F14 & TCTATAGAACGCAACATAGCATAAAAG & & & \\
\hline
\end{tabular}

final extension $72^{\circ} \mathrm{C}$ for $3 \mathrm{~min}$ and $4^{\circ} \mathrm{C}$ hold. The bands were gel-eluted corresponding to different products and pooled for NGS library construction (Fig. 1).

\section{NGS library construction, sequencing and assembly}

The pooled PCR products were sheared to smaller sizes using Bioruptor. One of each of the Ion Torrent and Illumina library was constructed per manufacturers' protocols. Briefly, PCR products were sonicated, adapter ligated and amplified for $\mathrm{x}$ cycles to generate a library. The libraries were sequenced to generate $14 \mathrm{k}$ reads of an average of $150 \mathrm{nt}$ SE reads on Ion Torrent, and 1.3 million reads of $72 \mathrm{nt}$ SE reads on Illumina GAIIx. High quality and vector filtered reads from Ion Torrent and Illumina sequencing were assembled (hybridassembly) using Mira-3.9.15 (http://sourceforge.net/apps/mediawiki/mira-assembler). The hybrid assembly generated 776 contigs. All 776 contigs were then used as input for CAP3 assembler which generated 38 contigs. The contigs were further filtered to remove short and duplicate contigs. Finally, only 14 contigs were retained and ORF prediction was carried out using ORF Finder (Open Reading Frame Finder) (http://www.ncbi.nlm.nih. gov/gorf/gorf.html). The schematic outline of the assembly is depicted in Fig. 2.

A manual examination of the 14 contigs revealed overlaps amongst all of them (except C30) (Fig. 2) and in collinear arrangement when compared with the F. hepatica mitochondrial sequence. The 14 contigs were manually joined wherever overlaps (minimum overlap $>5$ ) were found and that resulted in two individual contigs which, in turn, were assembled into one single contig with the addition of a couple of Ns. To resolve the remaining gaps between the two contigs as well as to confirm the assembly both the regions were amplified and Sanger sequenced. The Sanger sequencing was carried out by designing two primers for both the contigs flanking the Ns to resolve this gap and to verify the assembly as well as closure of the gap that was remaining after joining the contigs 


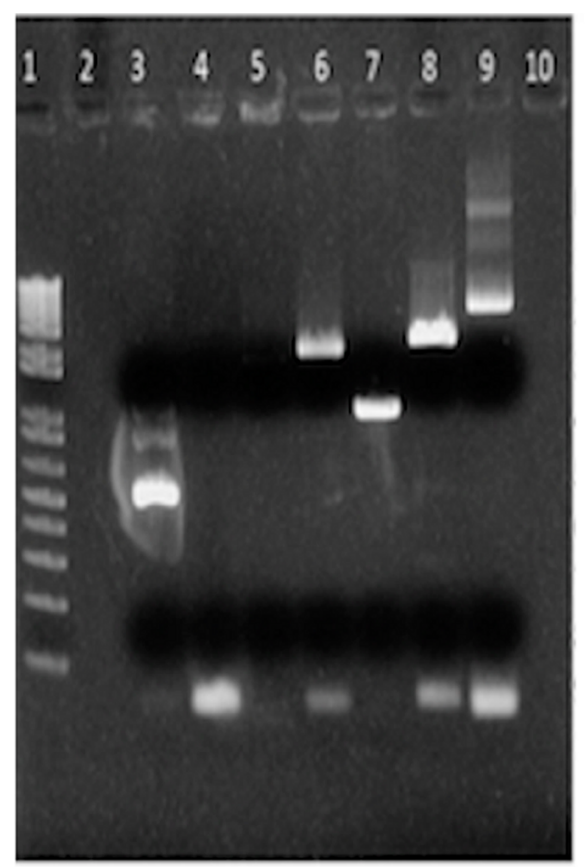

A

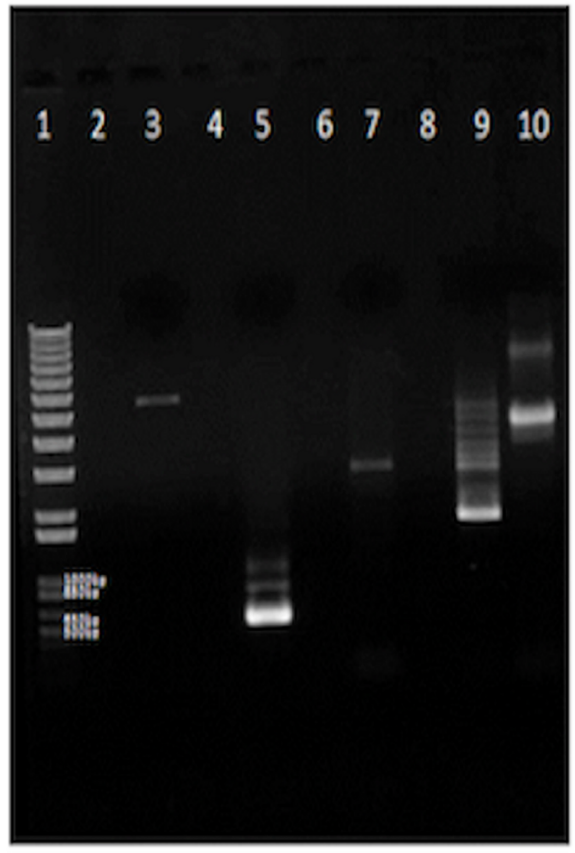

B

Figure 1 Gel images of the long range PCR products. Long-range PCR carried out using 10 ng of genomic DNA from F. buski (FD2 and FD3 samples). Gel-eluted bands corresponding to different products that were pooled for NGS library construction are shown. (A) SO_1625_mt gel (elution). Lane Order: Lane 1-1 kb plus ladder, Lane 2-Blank, Lane 3-P1 primer pair, Lane 4-P2 primer pair, Lane 5-P3 primer pair, Lane 6-P4 primer pair, Lane 7-P5 primer pair, Lane 8-P6 primer pair, Lane 9-P7 primer pair, Lane10-Blank. Lanes 3, 5, 6, 7, 8, and 9 (both bands) were taken for Ion Torrent Library Prep. (B) SO_1625_mt gel (elution). Lane Order: Lane 1-1 kb plus ladder, Lane 2-Blank, Lane 3-P7 + P2 primer pair, Lane 4-Blank, Lane 5-P1 primer pair, Lane 6-Blank, Lane 7-P2 primer pair, Lane 8-Blank, Lane 9-P3 primer pair, Lane 10-P7 primer pair. Lanes 3, 5 (all bands), 7, 9 (bright band) and Lane 10 (both bands) were taken for Ion Torrent Library Prep.

manually. The Sanger data in two regions was used to replace the NGS assembly-derived data to refine the assembly and obtain one single contig with no gaps. Region 1 was a $\sim 500$ nt overlapping region between C2 and C16. Region 2 was sequenced using one primer in C24 and the second primer in C26. Considering the finished mitochondrial genome, i.e., from position 1 to 14118, two primer pairs were designed as detailed below:

Set 1: fw primer position \# 7395-7414 (Length $=20$ )

FORWARD PRIMER: TGGTTATTCTGGTTGGGGAG

rev primer position \# 8137-8159 (Length $=23$ )

REVERSE PRIMER: AACCCTCCTATAAGAACCCAAAG $(\mathrm{RC}=)$ CTTTGGGTTCTTATAGGAGGGTT

The Sanger sequence data and NGS assembly aligned to each other with $94 \%$ identity. Twenty-nine out of 494 positions showed discordance between the Sanger sequencing and NGS-derived sequencing for this region (Fig. 3). These discordances consist of 19 


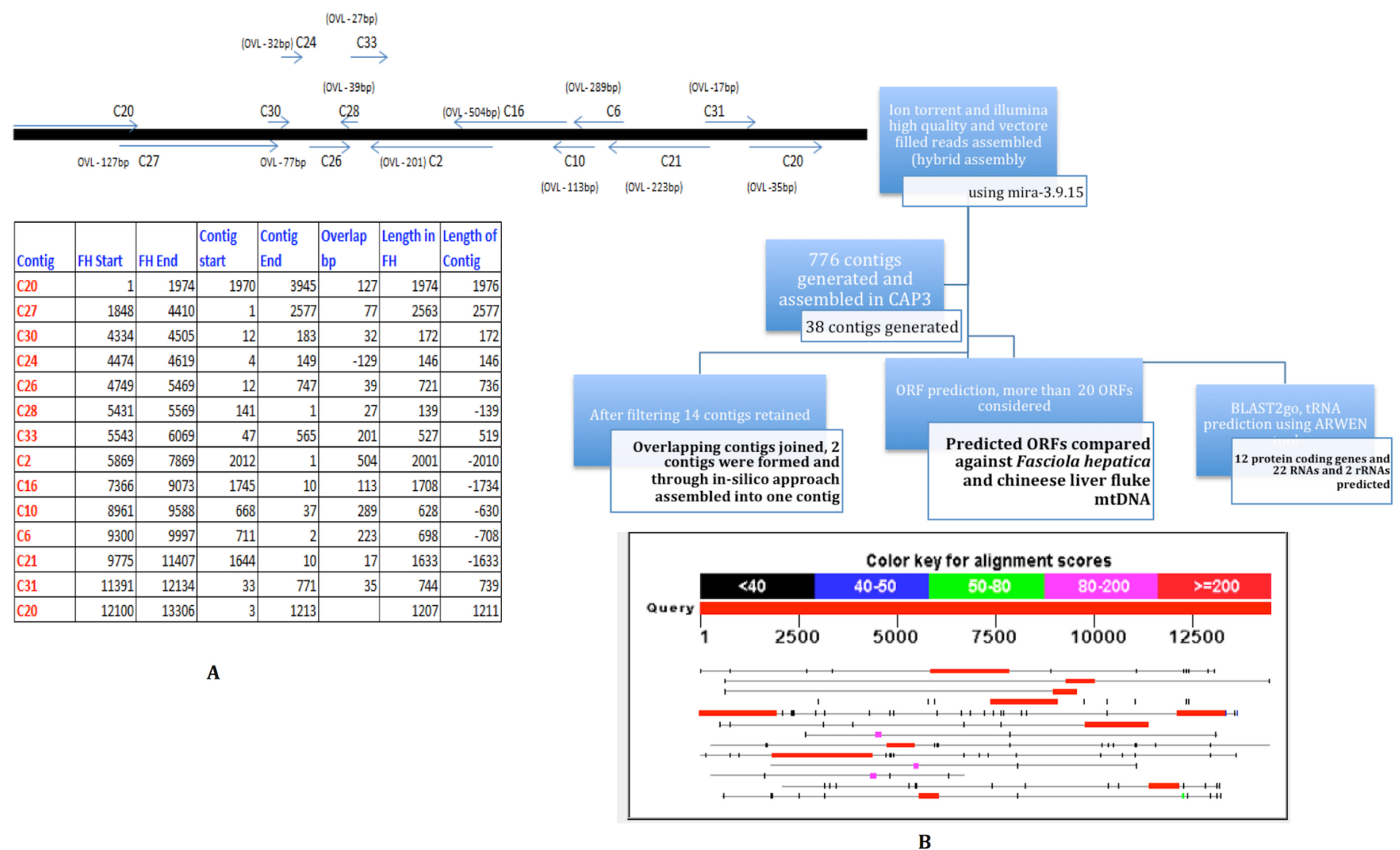

Figure 2 Strategy for MIRA and CAP3 Assembly for mtDNA NGS data. Ion Torrent and Illumina High quality and vector filtered reads assembled (hybrid assembly) using mira-3.9.15. 776 contigs were generated from the hybrid assembly. All the 776 contigs were fed in CAP3 assembler. Post filtering 14 contigs were retained. From 14 contigs overlapping contigs were joined and 2 contigs were formed, which were finally joined as one with the addition of couple of Ns. Predicted ORFs were compared against $F$. hepatica coding regions. Region 1 is a $\sim 500$ nt overlapping region between $\mathrm{C} 2$ and C16. Region 2 was sequenced using one primer in C24 and the second primer in C26.

gaps and 10 mismatches that can be introduced by either the sequencing chemistry (for e.g., homopolymeric stretches in Ion Torrent) or an assembly artifact (e.g., Ns). Overall, the Sanger sequencing confirmed the assembly pipeline and also corrected errors that are commonly observed in NGS pipelines.

Set 2: fw primer position \# 4634-4655 (Length $=22$ ),

FORWARD PRIMER: TAGGGTTATTGGTGTTAACCGG

reverse primer position \#4961-4937 (Length $=25$ )

REVERSE PRIMER: CAACAAACCAACAACTATACATCCC

REV PRIMER RC:- GGGATGTATAGTTGTTGGTTTGTTG

The region between contigs C24 and C26 did not show any overlap. The forward primer was $94 \mathrm{bp}$ inward from the junction on C24 and the reverse primer was $112 \mathrm{bp}$ outward from the junction on C26. The expected region based on assembly for contigs 24 and 26 and the Sanger results are shown in Fig. 4. The bases in brown colour within brackets are 


\begin{abstract}
TGGTTATTCTGGTTGGGGAGTTGATTTTTTGATGTTTTCTTTGCATTTGGCGGGGGTTTCTAGTATTTTTAGGTCTATTAAATTTATTTGTACTATAGTGGAAGTGATGTTTGAGGAAGGAACAGGACGTTTAA GTATTTAGTTTGAGCTTATTTGTTTACTTCTATTTTGTTGCTTTTGTCTTTGCCGGTTTGCTGCTGCTATAACANTTGTTGTTTGATCGTAGGTTTGGCTCTGCCTTTNGATCCNTATGGGGGAGATCCAGT GTTATTTCAGCATTTGTTTTGGTTTTTTGGGCATCCGGAGGTTTATGTTTTGATTTTGCCGGGGTTTGGTGTTATTAGACATATTTGTGTAACTTTAACTAATAAAGATTCTTTGTTTGGTTATTATGGTCTTC TTTTGGCCATGGCTGCGATTNGTTTGTTTGGGTAGTATTGTGTGGGCTCACCATATTGTTTATGGTTNGGTTTAGATGTTCATANCTGCGGTNTTTTTNAGTTCTNGTTACTATGGTGATTAGTATACCTACA GGGATTAAGGTTTTTTCTTGGTTGATTATGTTGGGGGGAGGTAGTTTGGTTCGTATTTGAGATCCGGTAGTGTGGTGAATTATAGGGTTTATTGTGTTATTTACTATAGGAGGTGTTACTGGTATAATGCTTTC

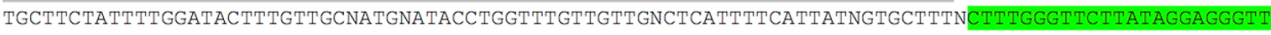

SANGER SEQUENCE IN FASTA FORMAT

$>0613$ 038_001_C2-C16_EHC2-E11.ab1 (QUERY)

ATTTGGCGGGGGTTTCTAGTATTTTAGGTCTATTAAATTATTTGTACTATAGTGGAAGTGATGTTTGAGGAAGGAACAGGACGTTTAAGTATTTTAGTTTGAGCTTATTTGTTACTTCTATTTTGTTGCTI TTGTCTTTGCCGGTTTGGCTGCTGCTATAACAATGTTGTTGTTTGATCGTAGGTTTGGCTCTGCCTTTTTTGATCCTATGGGGGGCGAGATCCAGTGTTATTTCAGCATTTGTTTGGTTTTTGGGCATCC GGAGGTTTATGTTTTGATTTTGCCGGGGTTTGGTGTTATTAGACATATTTGTGTAACTTTAACTAATAAAGATTCTTTGTGTGGTTATTGTGGTCTTGTTTTGGGATGGCTGCGATTGTTTGTTTGGGTAGTAT TGTGTGGGCTCACCAGATGTTTATGGTTGGTTAGATGTTCATACTGCGGTTTTTTTAGTTCTGTTACGATGGTGATGAC

$>$ NGS_ASSEMBLY (SUBJECT)

ATTTGGCGGGGTTTCTAGTATTITAGGTCTATTAAATTTATTTGTACTATAGTGGAAGTGATGTTIGAGGAAGGAACAGGACGTTTAAGTATTAGTTMAGCTTATTTGTTACTTCTATTTTGTTGCTTI TGTCTTTGCCGGTTGGCTGCTGCTATAACANTTGTTGTTTGATCGTAGGTTTGGCTCTGCCTTTNGATCCNTATGGGGGGAGATCCAGTGTTATTTCAGCATTTGTTTTGGTTTTTGGGCATCCGGAGGTTI ATGTTTTGATTTTGCCGGGGTTTGGTGTTATTAGACATATTTGTGTAACTTTAACTAATAAAGATTCTTTGTTTGGTTATTATGGTCTTGTTTTGGCCATGGCTGCGATTNGTTTGTTTGGGTAGTATTGTGTG GGCTCACCATATTGTTTATGGTTNGGTTTAGATGTTCATANCTGCGGTNTTTTTTNAGTTCTNGTTACTATGGTGATTAGTATACCTACAGGGATTAAGGTTTTTTCTTGGTTGATTATGTTGGGGGGAGGTAG TTTGGTTCGTATTTGAGATCCGGTAGTG

A

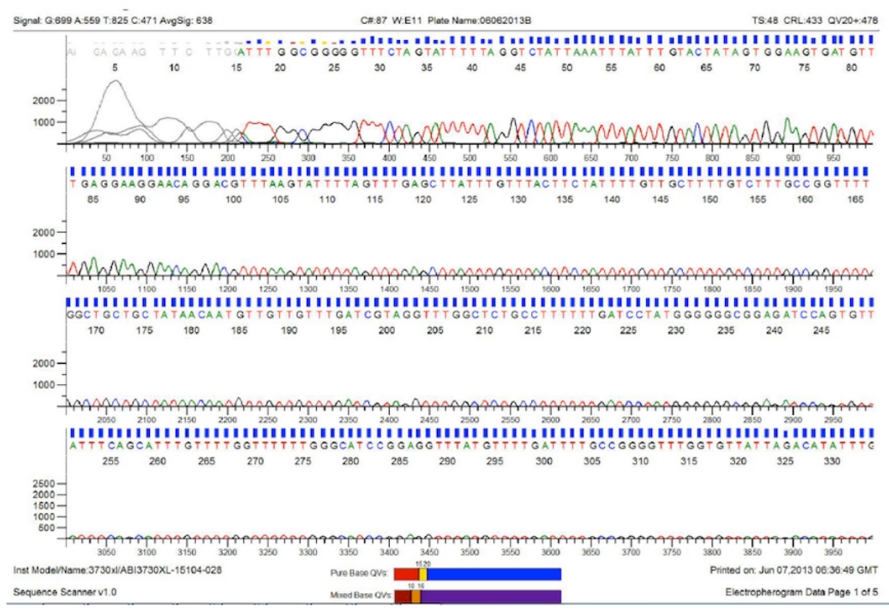

B

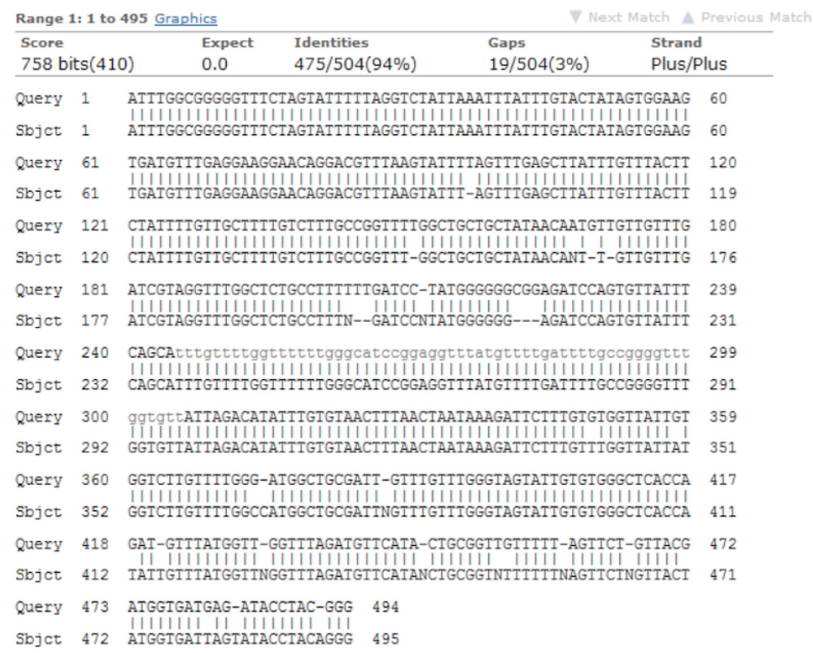

C

Figure 3 Assembly confirmation of the $\sim 500$ nucleotide region between C2-C16. Primers spanning the 500 bp overlap junction between contig 2 and contig 16 are marked in green. Sanger sequenced region (query) and NGS assembly (subject) were aligned with $94 \%$ identity with strong supportive E-values (0.0). Twenty nine out of 494 positions showed discordance between the Sanger sequencing and NGS-derived sequencing consisting of 19 gaps and 10 mismatches that may be introduced by either sequencing chemistry (e.g., homopolymeric stretches in Ion Torrent) or an assembly artifact (e.g., Ns). 
$>$ CONTIG24------

AGgTTGTGTTCTAATTGATTAGTTATATGGTCTTTGTCTACTTTTTTGAAGGCTCCTGTTTTTTTT СTTCСTTTCTTTTGTCAGCGGGTGGTTATTTTTTGTGAATATTCTGTGTTGTGTAGGGTTATTG GTGTTAACCGGCTTGTTTGGTTTATAGATTTGGTGGGTTTATTGTTGATGTCATATGATGTTG TCTTCTAGGGCGGGTTT

$>$ Contig26

GGTGTGTGATCTTATTTTGTTTTTGTTTCTTTTGCTTTCTCTTCCGGTTTCTTTTCTTTGTTT TATAAGTTGGTTATGGTTTTTGGGATGTATAGTTGTTGGTTTTTG

>0613_038_003_C24-C26_FHC24-G11.ab1 (SANGER PASS BASES ARE IN BOLD)

GGTTGGGTTTATTGTTGATGTCATATGATGTTGTCTTCTAGGGCGGTTT (TAGTTGCTATGTGTTT TGTTTTATCTTCTGATGTGATGTTTTTTGTTTTTTTGTTTATTGTTTTTGAGCTTCTTTGGTGAT TTTGTTTTTGAGGAATTGTAGCGTTAGGGGTTTAGATGGG) GGTGTGTGATCTTATTTTGTTTTTT GTTTTСTTTTGCTTTСTСTTCСGGTTTСTTTTTСTTTGTTTTATAAGTTGGTTATGGTTTTTC

Figure 4 Assembly confirmation for the C24-C26 region. Region between contigs C24 and C26 showing no overlap regions. Forward primer is $94 \mathrm{bp}$ inward from the junction on $\mathrm{C} 24$ and the reverse primer 112 bp outward from the junction on C26. The bases in brown colour in brackets are those that fill the gap between $\mathrm{C} 24$ and $\mathrm{C} 26$.

The data pertaining to this study is available in the National Centre for Biotechnology Information (NCBI) Bioproject database with Accession: PRJNA210017and ID: 210017. The contig assembly files are deposited in NCBI Sequence Read Archive (SRA) with Accession: SRR924085.

\section{In silico analysis for nucleotide sequence statistics, protein cod- ing genes (PCGs) prediction, annotation and tRNA prediction}

Sequences were assembled and edited both manually and using CLC Genome Workbench V.6.02 with comparison to published flatworm genomes. The platyhelminth genetic code (Telford et al., 2000) was used for translation of reading frames. Protein-coding genes were identified by similarity of inferred amino acid sequences to those of other platyhelminth mtDNAs available in GenBank. Boundaries of rRNA genes both large (rrnL) and small (rrnS) were determined by comparing alignments and secondary structures with other known flatworm sequences. The program ARWEN (Laslett \& Canbäck, 2008) was used to identify the tRNA genes (trns). To find all tRNAs, searches were modified to find secondary structures occasionally with very low Cove scores $(<0.5)$ and, where necessary, also by restricting searches to find tRNAs lacking DHU arms (using the nematode tRNA option). Nucleotide codon usage for each protein-encoding gene was determined using the program Codon Usage) at http://www.bioinformatics.org/sms2/codon_usage. html. The ORFs and codon usage profiles of PCGs were analyzed. Gene annotation, genome organization, translation initiation, translation termination codons, and the 


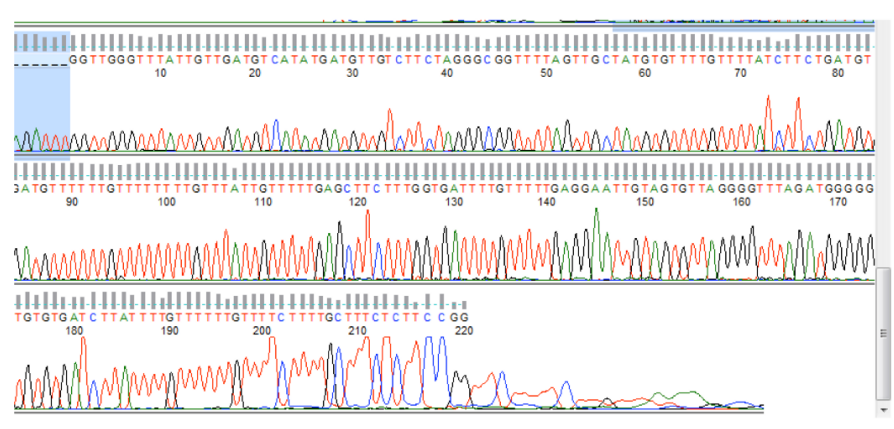

>FD3_C24-C26_REV (RC)

GGTTGGGTTTATT'GTTGATGTCATATGATGTTGTCTTCTAGGGCGGTTT TAGTTGCTATGTGTTTTGTTTTATCTTCTGATGTGATGTTTTTTGTTTT TTTTGTTTATTGTTTTTGAGCTTCTTTGGTGATTTTGTTTTTGAGGAAT TGTAGTGTTAGGGGTTTAGATGGGGGTGTGTGATCTTATTTTGTTTTTT GTTTTCTTTTGCTTTCTCTTCCGG

\begin{tabular}{lllll}
\multicolumn{2}{l}{ Range 1: 1 to 220} & \multicolumn{3}{c}{ Graphics } \\
\hline Score & Expect & Identities & Gaps & Strand \\
401 bits(217) & $6 \mathrm{e}-117$ & $219 / 220(99 \%)$ & $0 / 220(0 \%)$ & Plus/Plus \\
\hline
\end{tabular}

\section{Query 1 GGITGGGITTATTGITGATGICATATGATGITGICTICTAGGGCGgttttagttgctatg 60}

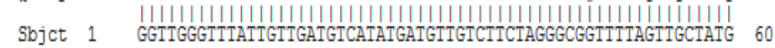

Query 61 tgttttgttttatcttctgatgtgatgttttttgtttttttgtttattgttttgagct 120

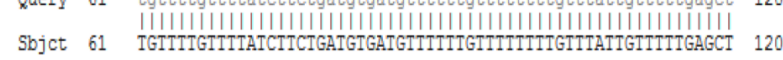

Query 121 tctttggtgattttgtttttgAGGAMTTGTAGTGTTAGGGGTTAAGATGGGGGTGIGIGA 180

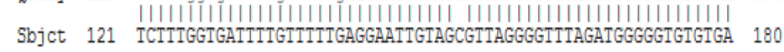

Query 181 TCTIAtttgtttttgtttctttgctttCTCTICCGG 220

Sbjet 181 ICTTATTTGITTTITGTTTCTTTGCTTICTCTICCGg 220

$\mathbf{A}$
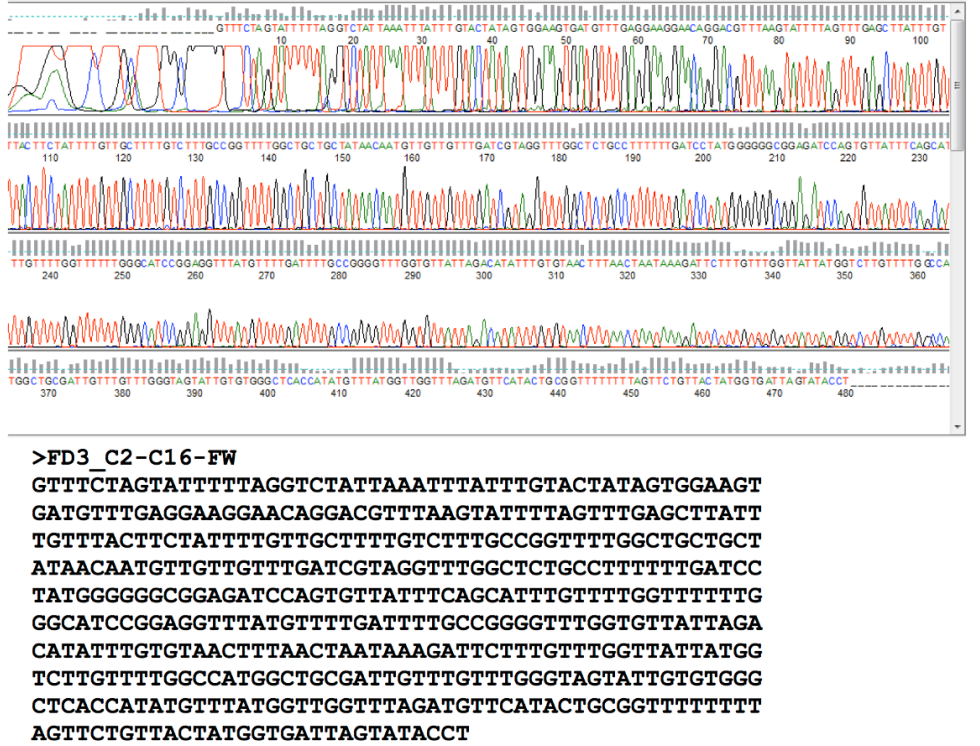

B

Figure 5 Sanger sequencing confirmatory results for FD2 and FD3 replicate samples. Two separate regions from two independent biological samples sequenced by Sanger methods showing 98-99\% identity between samples FD2 (subject) and FD3 (query) in the regions C2-C16 and C24-C26. 


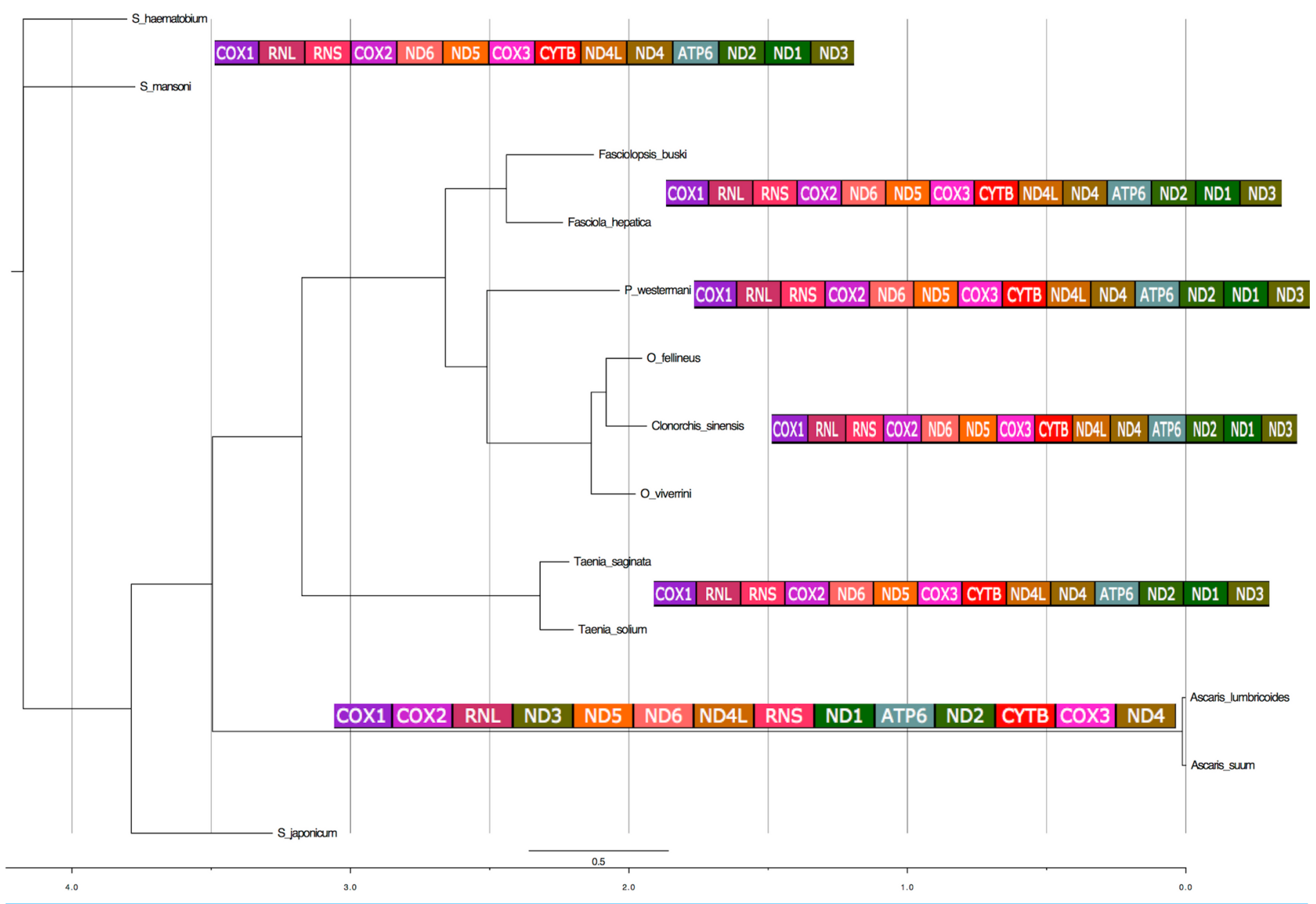

Figure 6 Phylogenetic analysis of the concatenated 12 protein coding genes from the platyhelminth mtDNA. Differences in the gene order in the mitochondrial genomes of parasitic flatworms from the Trematoda and Cestoda and taking Nematoda (Ascaridida) as an outgroup. Phylogenetic analyses of concatenated nucleotide sequence datasets for all 12 PCGs were performed using Bayesian Inference using four MCMC chains and 106 generations, sampled every 1,000 generations. Bayesian posterior probability (BPP) values were determined after discarding the initial 200 trees (the first $2 \times 105$ generations) as burn-in. Using the phylogeny estimated from the nuclear ribosomal DNA data set, pictograms of full mitochondrial genes are indicated next to the individual leaves of the tree. $x$-axis represents substitution rates per unit. 

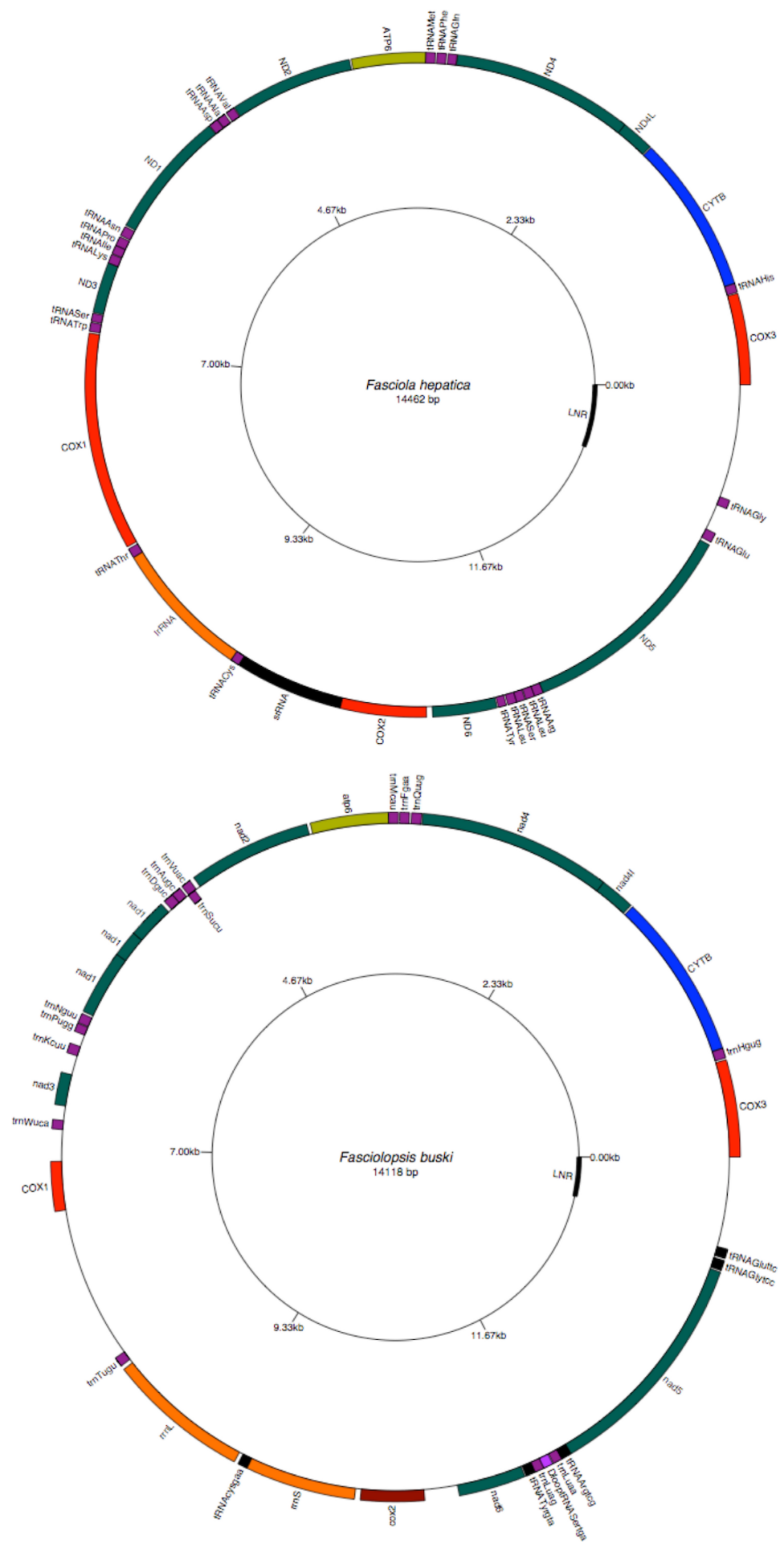

A

B

Figure 7 Circular genome map of Fasciola hepatica and Fasciolopsis buski. The manual and in silico annotations with appropriate regions for F. buski (7A) and annotated GenBank flat file for F. hepatica (7B) were drawn into a circular graph in GenomeVX depicting the 12 PCGs and 22tRNAs. 


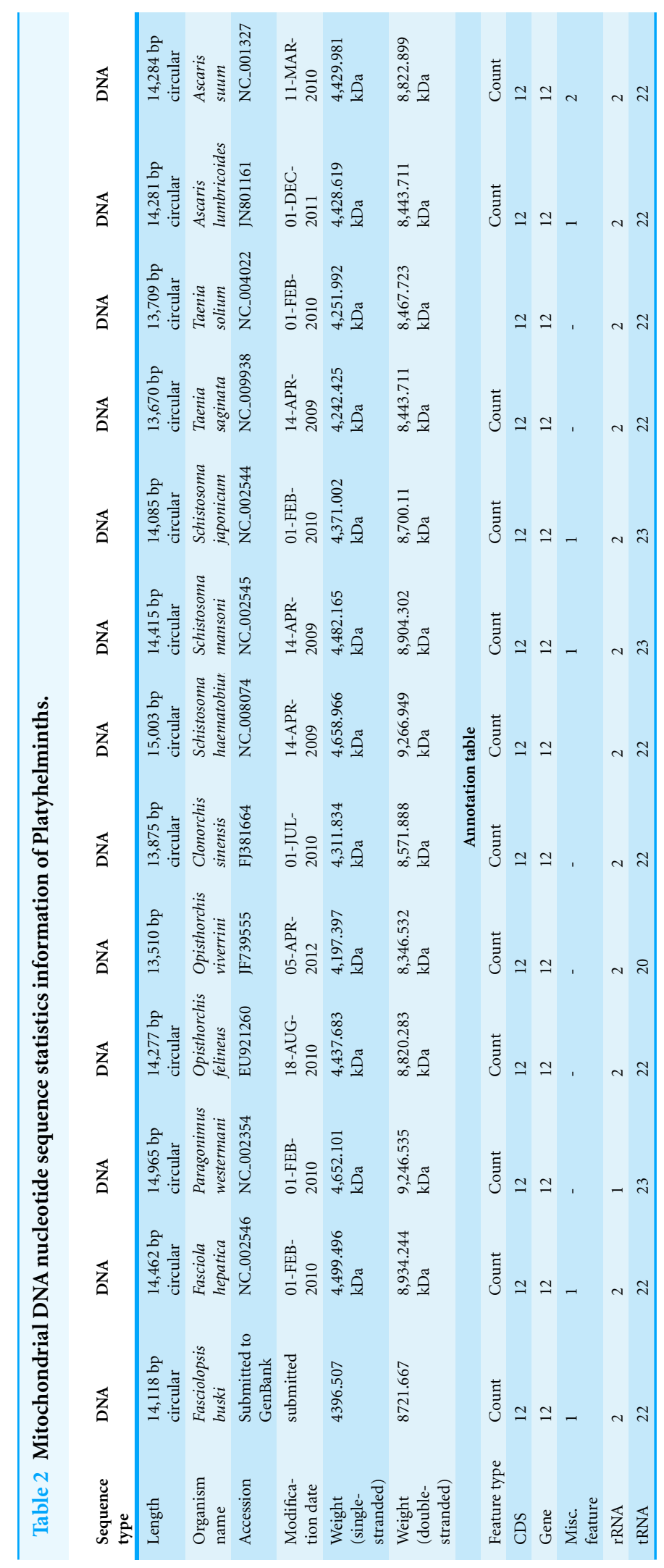


Table 3 Atomic composition and nucleotide distribution table of Fasciolopsis buski mtDNA. Ambiguous residues are omitted in atom counts.

\begin{tabular}{|c|c|c|c|c|c|}
\hline Atom & Count & Frequency & Nucleotide & Count & Frequency \\
\hline \multicolumn{3}{|c|}{ As single stranded } & Adenine (A) & 2509 & 0.178 \\
\hline Hydrogen $(\mathrm{H})$ & 174924 & 0.376 & Cytosine (C) & 1281 & 0.091 \\
\hline Carbon $(\mathrm{C})$ & 139209 & 0.299 & Guanine $(\mathrm{G})$ & 3925 & 0.278 \\
\hline Nitrogen $(\mathrm{N})$ & 48681 & 0.105 & Thymine (T) & 6334 & 0.449 \\
\hline Oxygen $(\mathrm{O})$ & 88120 & 0.19 & Purine (R) & 0 & 0 \\
\hline Phosphorus (P) & 14049 & 0.03 & Pyrimidine (Y) & 0 & 0 \\
\hline \multicolumn{3}{|c|}{ As double stranded } & Adenine or cytosine (M) & 0 & 0 \\
\hline Hydrogen $(\mathrm{H})$ & 346023 & 0.375 & Guanine or thymine $(\mathrm{K})$ & 0 & 0 \\
\hline Carbon $(\mathrm{C})$ & 275774 & 0.299 & Cytosine or guanine (S) & 0 & 0 \\
\hline Nitrogen $(\mathrm{N})$ & 103549 & 0.112 & Adenine or thymine (W) & 0 & 0 \\
\hline Oxygen $(\mathrm{O})$ & 168590 & 0.183 & Not adenine (B) & 0 & 0 \\
\hline \multirow[t]{6}{*}{ Phosphorus (P) } & 28098 & 0.03 & Not cytosine (D) & 0 & 0 \\
\hline & & & Not guanine $(\mathrm{H})$ & 0 & 0 \\
\hline & & & Not thymine (V) & 0 & 0 \\
\hline & & & Any nucleotide (N) & 69 & 0.005 \\
\hline & & & $\mathrm{C}+\mathrm{G}$ & 5206 & 0.369 \\
\hline & & & $A+T$ & 8843 & 0.626 \\
\hline
\end{tabular}

\section{RESULTS AND DISCUSSION}

\section{Gene contents and organization}

The intestinal fluke F. buski has a $\mathrm{mt}$ genome typical of most platyhelminths (Fig. 7A). The circular genome consists of $14118 \mathrm{nt}$ bp and is almost similar to that of Fasciola hepatica (Fig. 7B). The 12 protein-coding genes fall into the following categories: nicotinamide dehydrogenase complex (nad1-nad6 and nad4L subunits); cytochrome c oxidase complex ( $\operatorname{cox} 1-\operatorname{cox} 3$ subunits); cytochrome b (cob) and adenosine triphosphatase subunit 6 (atp6). Two genes encoding ribosomal RNA subunits are present: the large subunit ( $\mathrm{rrnL}$ or $16 \mathrm{~S}$ ) and small subunit (rrnS or 12S), which are separated by trnC, encoding the transfer RNA (tRNA) for cysteine. As in other mt genomes, there are 22 tRNA genes, denoted in the figure by the one-letter code for the amino acid they encode. Leu and Ser are each specified by two different tRNAs, reflecting the number and base composition of the relevant codons. As in other flatworms, all genes are transcribed in the same direction (Fig. 7). Genes lack introns and are usually adjacent to one another or separated by only a few nucleotides. However, some genes overlap, most notably nad4, nad4L and with regions of the long non coding region, which is almost $500 \mathrm{nt}$ length.

\section{Nucleotide composition and codon usage}

Invertebrate mt genomes tend to be AT-rich (Malakhov, 1994), which is a notable feature in PCGs of several parasitic flatworms. However, nucleotide composition is not uniform among the species (Table 2). Values for $>70 \%$ AT are seen in all Schistosoma spp. except for S. mansoni (68.7\%), whereas F. buski and Fasciola hepatica are 60\% AT rich and 
Table 4 Codon usage for $\boldsymbol{F}$. buski mtDNA genome.

\begin{tabular}{|c|c|c|c|c|}
\hline AmAcid & Codon & Number & $/ 1000$ & Fraction \\
\hline Ala & GCG & 22.00 & 4.74 & 0.20 \\
\hline Ala & GCA & 26.00 & 5.60 & 0.24 \\
\hline Ala & GCT & 46.00 & 9.91 & 0.43 \\
\hline Ala & GCC & 14.00 & 3.02 & 0.13 \\
\hline Cys & TGT & 239.00 & 51.49 & 0.80 \\
\hline Cys & TGC & 58.00 & 12.49 & 0.20 \\
\hline Asp & GAT & 90.00 & 19.39 & 0.85 \\
\hline Asp & GAC & 16.00 & 3.45 & 0.15 \\
\hline Glu & GAG & 63.00 & 13.57 & 0.68 \\
\hline Glu & GAA & 30.00 & 6.46 & 0.32 \\
\hline Phe & TTT & 442.00 & 95.22 & 0.85 \\
\hline Phe & TTC & 79.00 & 17.02 & 0.15 \\
\hline Gly & GGG & 119.00 & 25.64 & 0.28 \\
\hline Gly & GGA & 67.00 & 14.43 & 0.16 \\
\hline Gly & GGT & 201.00 & 43.30 & 0.48 \\
\hline Gly & GGC & 31.00 & 6.68 & 0.07 \\
\hline His & CAT & 19.00 & 4.09 & 0.73 \\
\hline His & CAC & 7.00 & 1.51 & 0.27 \\
\hline Ile & ATT & 168.00 & 36.19 & 0.86 \\
\hline Ile & ATC & 28.00 & 6.03 & 0.14 \\
\hline Lys & AAG & 42.00 & 9.05 & 1.00 \\
\hline Leu & TTG & 263.00 & 56.66 & 0.36 \\
\hline Leu & TTA & 193.00 & 41.58 & 0.27 \\
\hline Leu & CTG & 70.00 & 15.08 & 0.10 \\
\hline Leu & CTA & 53.00 & 11.42 & 0.07 \\
\hline Leu & CTT & 117.00 & 25.20 & 0.16 \\
\hline Leu & СТC & 26.00 & 5.60 & 0.04 \\
\hline Met & ATG & 101.00 & 21.76 & 0.58 \\
\hline Met & ATA & 72.00 & 15.51 & 0.42 \\
\hline Asn & AAA & 39.00 & 8.40 & 0.34 \\
\hline Asn & AAT & 64.00 & 13.79 & 0.56 \\
\hline Asn & AAC & 11.00 & 2.37 & 0.10 \\
\hline Pro & CCG & 16.00 & 3.45 & 0.27 \\
\hline Pro & CCA & 10.00 & 2.15 & 0.17 \\
\hline Pro & CCT & 27.00 & 5.82 & 0.45 \\
\hline Pro & CCC & 7.00 & 1.51 & 0.12 \\
\hline Gln & CAG & 19.00 & 4.09 & 0.63 \\
\hline Gln & CAA & 11.00 & 2.37 & 0.37 \\
\hline Arg & CGG & 34.00 & 7.32 & 0.33 \\
\hline Arg & CGA & 15.00 & 3.23 & 0.14 \\
\hline Arg & CGT & 44.00 & 9.48 & 0.42 \\
\hline Arg & CGC & 11.00 & 2.37 & 0.11 \\
\hline Ser & AGG & 100.00 & 21.54 & 0.25 \\
\hline
\end{tabular}




\begin{tabular}{|c|c|c|c|c|}
\hline AmAcid & Codon & Number & $/ 1000$ & Fraction \\
\hline Ser & AGA & 49.00 & 10.56 & 0.12 \\
\hline Ser & AGT & 88.00 & 18.96 & 0.22 \\
\hline Ser & AGC & 17.00 & 3.66 & 0.04 \\
\hline Ser & TCG & 30.00 & 6.46 & 0.07 \\
\hline Ser & TCA & 25.00 & 5.39 & 0.06 \\
\hline Ser & TCT & 69.00 & 14.86 & 0.17 \\
\hline Ser & TCC & 28.00 & 6.03 & 0.07 \\
\hline Thr & ACG & 7.00 & 1.51 & 0.14 \\
\hline Thr & ACA & 14.00 & 3.02 & 0.27 \\
\hline Thr & ACT & 19.00 & 4.09 & 0.37 \\
\hline Thr & ACC & 11.00 & 2.37 & 0.22 \\
\hline Val & GTG & 114.00 & 24.56 & 0.22 \\
\hline Val & GTA & 95.00 & 20.47 & 0.19 \\
\hline Val & GTT & 270.00 & 58.16 & 0.53 \\
\hline Val & GTC & 34.00 & 7.32 & 0.07 \\
\hline $\operatorname{Trp}$ & TGG & 174.00 & 37.48 & 0.60 \\
\hline $\operatorname{Trp}$ & TGA & 115.00 & 24.77 & 0.40 \\
\hline Tyr & TAT & 160.00 & 34.47 & 0.83 \\
\hline Tyr & TAC & 32.00 & 6.89 & 0.17 \\
\hline End & TAG & 118.00 & 25.42 & 0.65 \\
\hline End & TAA & 63.00 & 13.57 & 0.35 \\
\hline \multicolumn{5}{|c|}{ Counts of di-nucleotides in F. buski mtDNA } \\
\hline 1.pos $\backslash 2 . p o s$ & A & C & G & $\mathbf{T}$ \\
\hline A & 467 & 191 & 766 & 1072 \\
\hline $\mathrm{C}$ & 217 & 204 & 262 & 593 \\
\hline G & 656 & 347 & 1263 & 1645 \\
\hline $\mathrm{T}$ & 1158 & 530 & 1619 & 2997 \\
\hline \multicolumn{5}{|c|}{ Frequency of di-nucleotides in F. buski mtDNA } \\
\hline 1.pos $\backslash 2$.pos & A & C & G & $\mathbf{T}$ \\
\hline A & 0.033 & 0.014 & 0.055 & 0.077 \\
\hline $\mathrm{C}$ & 0.016 & 0.015 & 0.019 & 0.042 \\
\hline G & 0.047 & 0.025 & 0.09 & 0.118 \\
\hline $\mathrm{T}$ & 0.083 & 0.038 & 0.116 & 0.214 \\
\hline
\end{tabular}

Paragonimus westermani, only 50\% AT rich. Cytosine is poorly represented in F. buski. The annotation and nucleotide sequence statistics are enumerated in Tables 2-5. The gene content and arrangement are identical to those of $F$. hepatica. ATG and GTG are used as the start-codons and TAG and TAA, the stop-codons.

Among species considerable differences in base composition in PCGs are reflected in differences in the protein sequences. However, the redundancy in the genetic code provides a means by which a mt genome could theoretically compensate for base-composition bias. Increased use of abundant bases in the (largely redundant) third codon position accounts for the fact that base composition bias would be less marked in the first and second codon positions. A phylogenetic tree was computed concatenating all the annotated 12 PCGs 
Table 5 mtDNA annotation of $\boldsymbol{F}$. buski and comparison with Fasciola hepatica.

\begin{tabular}{lllll} 
& Gene & $\begin{array}{l}\text { Length in } \\
\text { F. hepatica }\end{array}$ & $\begin{array}{l}\text { Gene prediction } \\
\text { length in } \text { F. buski }\end{array}$ & $\begin{array}{l}\text { \% of } \boldsymbol{F} \text {. hepatica } \\
\text { CDS covered in } \text { F. buski }\end{array}$ \\
\hline 1 & nad3 & 118 & 97 & 82.20 \\
2 & nad2 & 288 & 257 & 89.24 \\
3 & cox1 & 510 & 470 & 92.16 \\
\hline 4 & nad1 & 300 & 278 & 92.67 \\
\hline 5 & cox2 & 200 & 194 & 97.00 \\
\hline 6 & cox3 & 213 & 210 & 98.59 \\
7 & nad5 & 522 & 515 & 98.66 \\
\hline 8 & cob & 370 & 366 & 98.92 \\
\hline 9 & nad6 & 150 & 149 & 99.33 \\
\hline 10 & nad4L & 90 & 90 & 100.00 \\
11 & nad4 & 423 & 423 & 100.00 \\
12 & atp6 & 172 & 172 & 100.00 \\
\hline
\end{tabular}

Table 6 Transfer RNA (tRNA) annotations of the Fasciolopsis buski mtDNA.

\begin{tabular}{lllllll}
$\begin{array}{l}\text { Contig } \\
\text { start }\end{array}$ & $\begin{array}{l}\text { Contig } \\
\text { end }\end{array}$ & Length & tRNA ID & Codon & GC \% & $\begin{array}{l}\text { Single letter } \\
\text { symbol }\end{array}$ \\
\hline 657 & 722 & 66 & mtRNA-His & GUG & 30.3 & H \\
3358 & 3424 & 67 & mtRNA-Gln & UUG & 35.8 & Q \\
3438 & 3501 & 64 & mtRNA-Phe & GAA & 39.1 & F \\
3511 & 3576 & 66 & mtRNA-Met & CAU & 40.9 & M \\
5086 & 5150 & 65 & mtRNA-Val & UAC & 32.3 & V \\
\hline 5168 & 5233 & 66 & mtRNA-Ala & UGC & 40.9 & A \\
5233 & 5300 & 68 & mtRNA-Asp & GUC & 38.2 & D \\
6213 & 6277 & 65 & mtRNA-Asn & GUU & 41.5 & N \\
6282 & 6339 & 58 & TV-loop mtRNA-Pro & UGG & 31 & P \\
6356 & 6418 & 63 & mtRNA-Ile & GAU & 47.6 & I \\
6421 & 6487 & 67 & mtRNA-Lys & CUU & 38.8 & K \\
6859 & 6919 & 61 & D-loop mtRNA-Ser & GCU & 41 & S1 \\
6932 & 6994 & 63 & mtRNA-Trp & UCA & 34.9 & W \\
\hline 8577 & 8641 & 65 & mtRNA-Thr & UGU & 30.8 & T \\
\hline 642 & 9707 & 66 & mtRNA-Cys & GCA & 50 & C \\
11581 & 11646 & 66 & mtRNA-Tyr & GUA & 39.4 & Y \\
11645 & 11709 & 65 & mtRNA-Leu & UAG & 49.2 & L1 \\
11708 & 11772 & 65 & D-loop mtRNA-Ser & UGA & 36.9 & S2 \\
11778 & 11841 & 64 & mtRNA-Leu & UAA & 37.5 & L2 \\
11839 & 11910 & 72 & mtRNA-Arg & UCG & 37.5 & R \\
13483 & 13551 & 69 & mtRNA-Gly & UCC & 30.4 & G \\
13565 & 13628 & 64 & mtRNA-Glu & UUC & 43.8 & Q \\
\hline
\end{tabular}




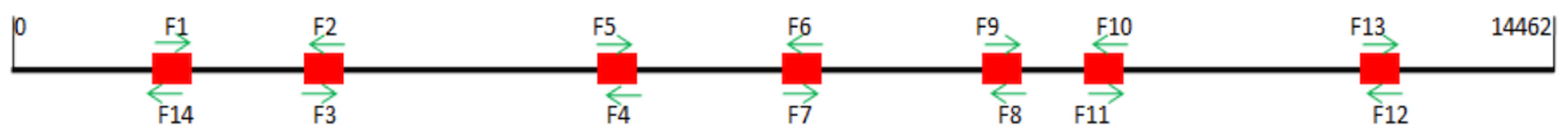

Alignment of Fascioopsis buski whole genome reads to the Fasciola hepatica mitochondrial genome

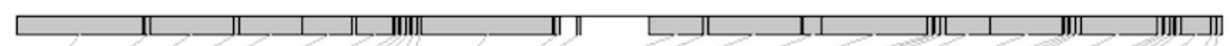

COX1 T RNL C RNS COX2 ND6 Y L S L2 R ND5 E G C0X3 H CYTB ND4L ND4 Q F M ATP6 ND2 Y A D ND1 N P I K ND3 S2 W

PLATYHELMINTHES Fasciola hepatica Proteins: 12 tRNAs: 22 rRNAs: 2 Length: 14462

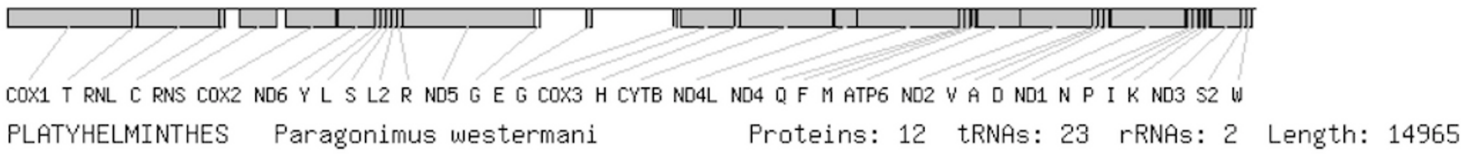

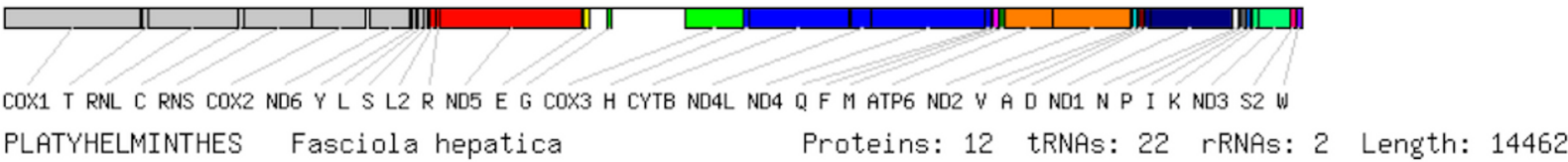
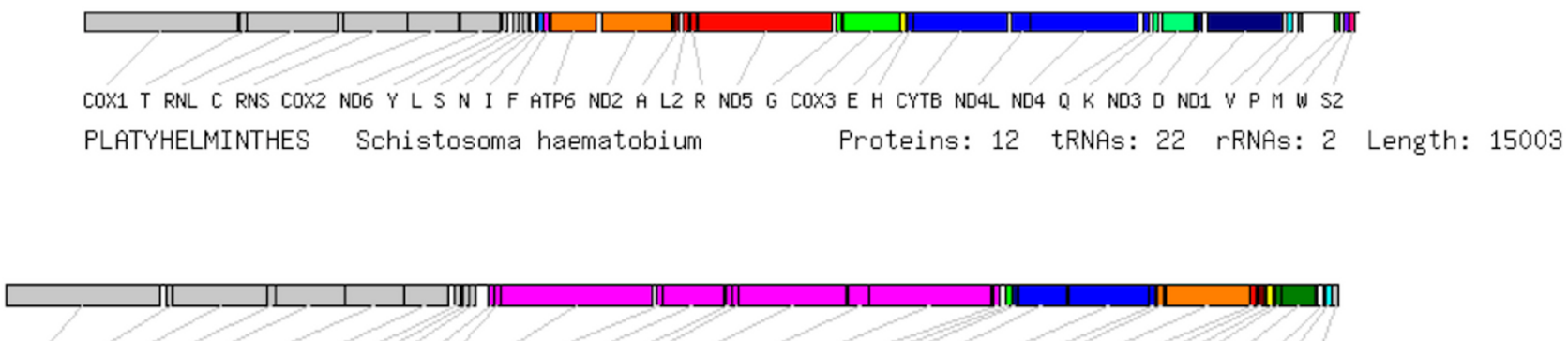

C0X1 T RNL C RNS C0X2 ND6 Y L S L2 R ND5 G C0X3 E H CYTB ND4L ND4 Q F MATP6 ND2 A D ND1 N P I K ND3 W V S2

PLATYHELMINTHES Schistosoma japonicum Proteins: 12 tRNAs: 22 rRNAs: 2 Length: 14085

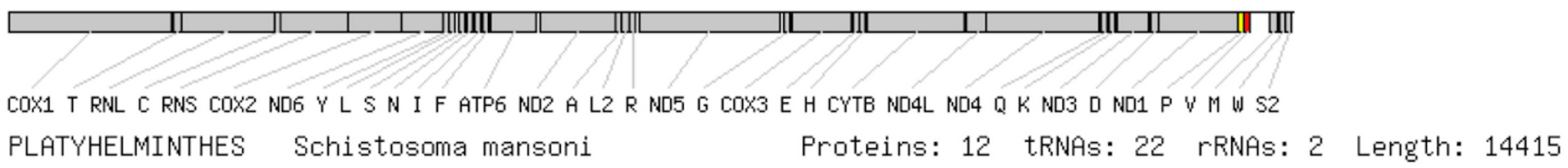

Figure 8 Synteny map of the representative species for the platyhelminth mtDNA. A comparative synteny for all the 12 protein coding genes and 22/23 tRNAs for the representative platyhelminth parasites (Schistosoma spp, F. buski, Fasciola hepatica, Paragonimus westermani).

that completely accounted for the platyhelminth phylogeny with the representative species (Fig. 8). F. buski came in the same clade with F. hepatica while Ascaris species formed the outgroup. The outgroup Ascaris lumbricoides displayed a different gene order that was aligned adjacent to the phylogenetic leaf nodes (Fig. 8). 


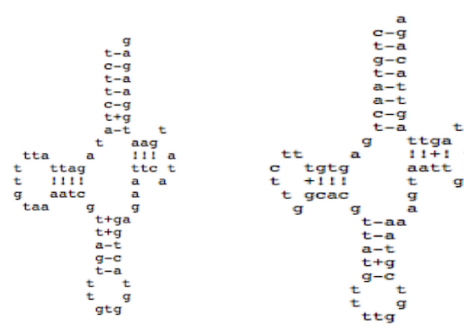

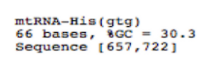

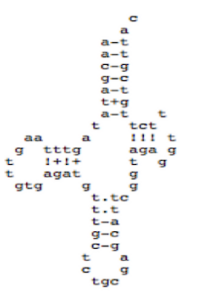

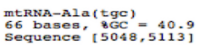

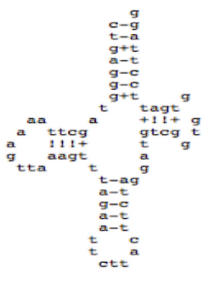

meRNA-LYB (Ctt)
67 baseg, 860 - 38.8
Sequence $[6301,6367]$

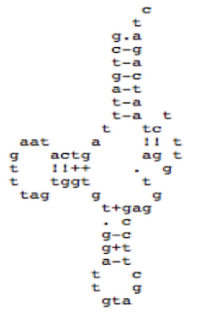

mtRkA-Tyr(gta)
66 bases, $86 \mathrm{GC}=39.4$
Sequence $[11462,11527]$

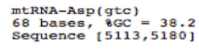

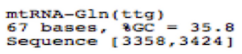
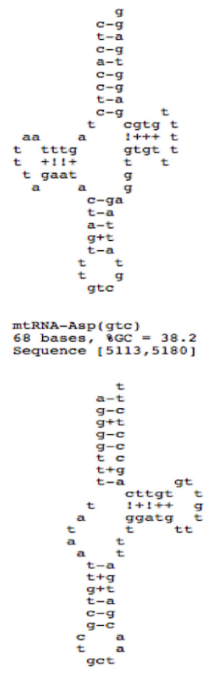

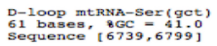

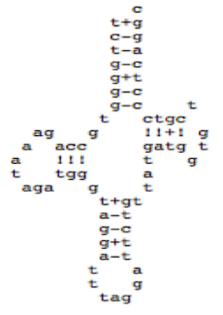

mtRMA-Leu (tag)
65 bases, $86 \mathrm{GC})=49.2$
Sequence $(11526,11590$

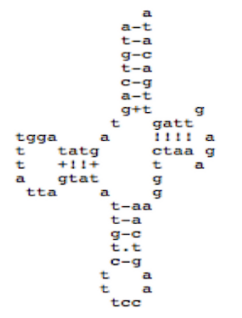

mtRRA-Gly $($ tCC $)$
69 bases, $8 G C)=30.4$
Sequence $[13364,13432]$

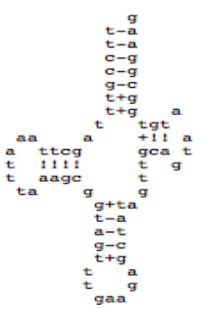

mtRRA-Phe (gaa)
64 bases, $8 \mathrm{gC})=39.1$
Sequence $\left[3438,350 i^{1}\right]$

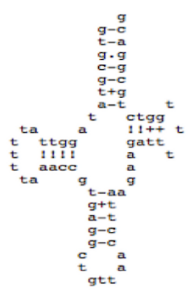

mtRrA-Asn (gtt)
65 bases, $860=41.5$
Sequence $[6093,61571$

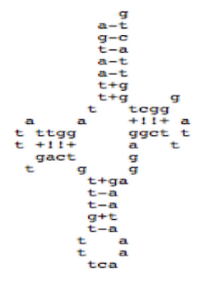

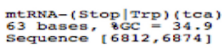

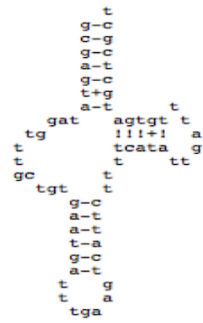

D-1oop mtrana-Ser (tga)
65 bases, $8 \mathrm{GC}-36.9)$
Sequence $\{11589,11653\}$

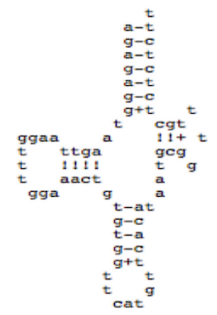

mtRWA-Met (cat)
66 bases, $8 G C=40.9$
Sequence $[3511,3576]$

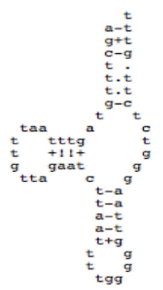

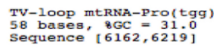

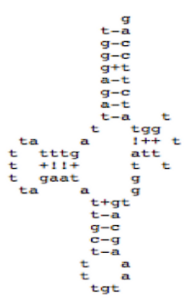

mtriA-Thr (tgt)
65 bases, $86 \mathrm{GC}-30.8$
Sequence $\{845,8522]$

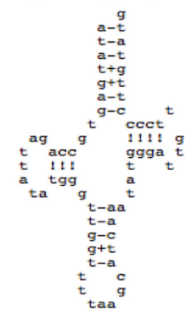

mtRkA-Leu $($ taa $)$
64 bases, $86 C=37.5$
Sequence $\{11659,11722\}$

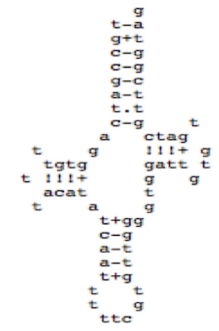

mtRNA-Glu (ttC)
64 bases, $86 C=43.8$
Sequence $[13446,13509]$

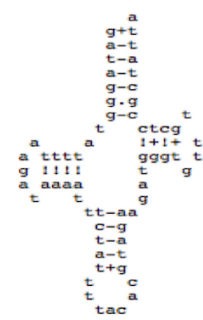

mtRNA-Val (tac)
65 bases, $86 c=32.3$
Sequence $[4966,5030]$

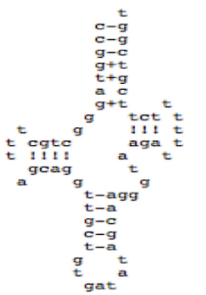

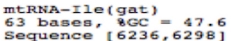

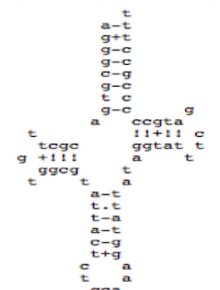

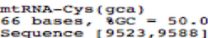

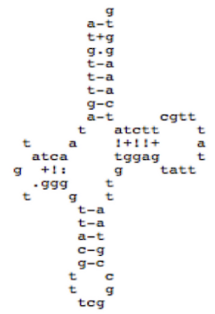

MtRRA-Arg(teg)
72 baseg, 8 BEC -37.5
Sequence $[11720,11791]$

Figure 922 tRNA secondary structures predicted using ARWEN. 


\section{Transfer and ribosomal RNA genes}

A total of 22 tRNAs were inferred along with structures (Fig. 9). The complete annotation along with their GC percentage is shown in Table 6. tRNA-Leu had the highest GC composition and the length varied between 60-70 nt bases. The tRNA genes generally resemble those of other invertebrates. A standard cloverleaf structure was inferred for most of the tRNAs. Exceptions include tRNA(S) in which the paired dihydrouridine (DHU) arm is missing as usual in all parasitic flatworm species (also seen in some other metazoans) and also tRNA(A) in which the paired DHU-arm is missing in cestodes but not in trematodes (and not usually in other metazoans) and hence, was also seen in F. buski. Structures for tRNA $(C)$ vary somewhat among the parasitic flatworms. A paired DHU-arm is present in F. buski, which is not seen in Schistosoma mekongi and cestodes. A comparative synteny for all the 12 protein coding genes and 22/23 tRNAs for the representative platyhelminth parasites can be seen across all the species under study (Fig. 8).

\section{CONCLUSIONS}

Although mt genomes of only a few parasitic flatworms have been sequenced, some general points can be made. The mtDNA of $F$. buski did not exhibit any surprising gene order composition or their organization relative to other invertebrates. As usual atp8 was absent, which is not without a precedent among invertebrates. Some typical secondary structures were inferred for some tRNA genes. Again, however, mt tRNA genes are less conserved in metazoans as compared to their nuclear counterparts. Gene order is similar or identical among most of the flatworms investigated, which might be expected for a taxon at this level of taxonomic heirarchy. In conclusion, the complete mtDNA sequences of $F$. buski will add to the knowledge of the trematode mitochondrial genomics and will aid in phylogenetic studies of the family Fasciolidae.

\section{ACKNOWLEDGEMENTS}

We would like to acknowledge M/s Genotypic Technologies, Bangalore, India for carrying out NGS sequencing for this project, especially the efforts of Mr. Rushiraj Manchiganti and Mr. Manoharan for the primer design strategy.

\section{ADDITIONAL INFORMATION AND DECLARATIONS}

\section{Funding}

The work was supported by the Department of Biotechnology, Government of India under the DBT-NER Twinning program sanctioned to VT, AB and DKB and partly by the Indian Council of Medical Research Project on worm zoonoses sanctioned to VT. The funders had no role in study design, data collection and analysis, decision to publish, or preparation of the manuscript. 


\section{Grant Disclosures}

The following grant information was disclosed by the authors:

Department of Biotechnology, Govt. of India: BT/48/NE/TBP/2010 dated March 14, 2011. Indian Council of Medical Research, Govt. of India: ECD/ADHOC/4/2011-12 dated May $16,2011$.

\section{Competing Interests}

Nandita Mullapudi is an employee of M/s Genotypic Technologies.

\section{Author Contributions}

- Alok Bhattacharya and Veena Tandon conceived and designed the experiments, analyzed the data, contributed reagents/materials/analysis tools, wrote the paper.

- Devendra Kumar Biswal, Sudeep Ghatani, Jollin A. Shylla and Ranjana Sahu performed the experiments.

- Nandita Mullapudi performed the experiments, analyzed the data.

\section{DNA Deposition}

The following information was supplied regarding the deposition of DNA sequences: National Centre for Biotechnology Information (NCBI) Bioproject database: PRJNA210017 and ID: 210017.

NCBI Sequence Read Archive (SRA): SRR924085.

\section{REFERENCES}

Benson DA, Karsch-Mizrachi I, Lipman DJ, Ostell J, David L. 2005. Wheeler: GenBank. Nucleic Acids Research 33:D34-D38 DOI 10.1093/nar/gki063.

Catanese G, Manchado M, Infante C. 2010. Evolutionary relatedness of mackerels of the Catanese genus Scomber based on complete mitochondrial genomes: strong support to the recognition of Atlantic Scomber colias and Pacific Scomber japonicus as distinct species. Gene 452:35-43 DOI 10.1016/j.gene.2009.12.004.

Chen HX, Sundberg P, Norenburg JL, Sun SC. 2009. The complete mitochondrial genome of Cephalothrix simula (Iwata) (Nemertea: Palaeonemertea). Gene 442:8-17

DOI 10.1016/j.gene.2009.04.015.

D’Onorio de Meo P, D’Antonio M, Griggio F, Lupi R, Borsani M, Pavesi G, Castrignanò T, Pesole G, Gissi C. 2011. MitoZoa 2.0: a database resource and search tools for comparative and evolutionary analyses of mitochondrial genomes in Metazoa. Nucleic Acids Research 40:D1168-D1172 DOI 10.1093/nar/gkr1144.

Jameson D, Gibson AP, Hudelot C, Higgs PG. 2003. OGRe: a relational database for comparative analysis of mitochondrial genomes. Nucleic Acids Research 31:202-206 DOI 10.1093/nar/gkg077.

Jex AR, Littlewood DTJ, Gasser RB. 2010. Toward next-generation sequencing of mitochondrial genomes-focus on parasitic worms of animals and biotechnological implications. Biotechnology Advances 28:151-159 DOI 10.1016/j.biotechadv.2009.11.002. 
Langmead B, Trapnell C, Pop M, Salzberg SL. 2009. Ultrafast and memory-efficient alignment of short DNA sequences to the human genome. Genome Biology 10:R25

DOI 10.1186/gb-2009-10-3-r25.

Laslett D, Canbäck B. 2008. ARWEN, a program to detect tRNA genes in metazoan mitochondrial nucleotide sequences. Bioinformatics 24:172-175 DOI 10.1093/bioinformatics/btm573.

Le TH, Nguyen VD, Phan BU, Blair D, McManus DP. 2004. Case report: unusual presentation of Fasciolopsis buski in a Vietnamese child. Transactions of the Royal Society of Tropical Medicine and Hygiene 98:193-194 DOI 10.1016/S0035-9203(03)00022-1.

Le TH, Pearson MS, Blair D, Dai N, Zhang LH, McManus DP. 2002. Complete mitochondrial genomes confirm the distinctiveness of the horse-dog and sheep-dog strains of Echinococcus granulosus. Parasitology 124:97-112 DOI 10.1017/S0031182001008976.

Li MW, Lin RQ, Song HQ, Wu XY, Zhu XQ. 2008. The complete mitochondrial genomes for three Toxocara species of human and animal health significance. BMC Genomics 9:224 DOI 10.1186/1471-2164-9-224.

Maddison WP, Maddison DR. 2001. Mesquite: a modular system for evolutionary analysis. Available at http://mesquiteproject.org.

Malakhov VV. 1994. Hope WD, ed. Nematodes: structure, development, classification, and phylogeny. Washington/London: Smithsonian Institution Press.

Olson PD, Cribb TH, Tkach VV, Bray RA, Littlewood DT. 2003. Phylogeny and classification of the Digenea (Platyhelminthes: Trematoda). International Journal of Parasitology 33:733-755 DOI 10.1016/S0020-7519(03)00049-3.

Prasad PK, Tandon V, Chatterjee A, Bandyopadhyay S. 2007. PCR-based determination of internal transcribed spacer (ITS) regions of ribosomal DNA of giant intestinal fluke, Fasciolopsis buski (Lankester, 1857) Looss, 1899. Parasitology Research 101:1581-1587

DOI 10.1007/s00436-007-0680-y.

Ronquist F, Huelsenbeck JP. 2003. MRBAYES 3: Bayesian phylogenetic inference under mixed models. Bioinformatics 19:1572-1574 DOI 10.1093/bioinformatics/btg180.

Roy B, Tandon V. 1993. Morphological and microtopographical strain variations among Fasciolopsis buski originating from different geographical areas. Acta Parasitologica 38:72-77.

Sambrook J, Fitsch EF, Maniatis T. 1989. Molecular cloning: a laboratory manual. Cold Spring Harbor: Cold Spring Harbor Press.

Telford MJ, Herniou EA, Russell RB, Littlewood DTJ. 2000. Changes in mitochondrial genetic codes as phylogenetic characters: two examples from the flatworms. Proceedings of the National Academy of Sciences of the United States of America 97:11359-11364 DOI 10.1073/pnas.97.21.11359.

Wolstenholme DR. 1992. Animal mitochondrial DNA, structure and evolution. International Review of Cytology 141:173-216.

Wyman SK, Jansen RK, Boore JL. 2004. Automatic annotation of organellar genomes with DOGMA. Bioinformatics 20:3252-3255 DOI 10.1093/bioinformatics/bth352. 